\title{
LONGITUDINAL ASSESSMENT OF AXONAL DYSFUNCTION IN SYSTEMIC LUPUS ERYTHEMATOSUS
}

\author{
Renan Bazuco Frittoli ${ }^{\star \star}$, Danilo Rodrigues Pereira ${ }^{1}$, Ana Carolina Londe ${ }^{1}$, Leticia Rittner ${ }^{1}$, Simone Appenzeller ${ }^{1}$ \\ 1. Universidade Estadual de Campinas, Campinas (SP), Brazil. \\ *Corresponding author: renan.bazuco@gmail.com
}

\section{BACKGROUND}

Neuropsychiatric impairment in systemic lupus erythematosus (SLE) has gained focus and importance. Proton magnetic resonance spectroscopy ( ${ }^{1} \mathrm{H}-\mathrm{MRS}$ ) has been shown to be an important noninvasive tool to quantify neuronal loss in central nervous system disorders. The objective was to analyze during four times the presence of axonal dysfunction in SLE and to determine clinical, laboratory and treatment features associated with its occurrence.

\section{MATERIALS AN METHODS}

One hundred thirty-four consecutive SLE patients (median age 29 years, range 7-67) from the rheumatology outpatient unit and 102 healthy controls (median age 28 years, 5-60). All patients underwent four magnetic resonance imaging (MRI) exams with a minimum interval of 1 year. We performed multi voxel $1 \mathrm{H}$-MRS using point-resolved spectroscopy sequence over the superiorposterior region of the corpus callosum (3T Phillips scanner) and signals from $\mathrm{N}$-acetylaspartate compounds (NAA), cholinebased compounds (Cho); creatine containing compounds ( $\mathrm{Cr}$ ), glutamate and glutamine (GIX) were measured and metabolites/ $\mathrm{Cr}$ ratios were determined. A complete clinical, laboratory and neurological evaluation was performed in all subjects. Neurological manifestations were analyzed according to the ACR classification criteria. Mood and anxiety disorders were determined through Beck depression (BDI) and Beck anxiety inventories (BAI). Systemic lupus erythematosus patients were further assessed for clinical and laboratory SLE manifestations, disease activity [SLE Disease Activity Index (SLEDAI)], damage [Systemic Lupus International Collaborating Clinics/American College of Rheumatology Damage Index (SDI)] and current drug exposures. Data were compared by non-parametric tests.

\section{RESULTS}

A difference in $\mathrm{GI} / \mathrm{Cr}$ ratio over time by the Friedman test in SLE patients ( $p=0.00001)$ was observed; $G \mid x / C$ r ratio correlated with illness time ( $r=-0.250 ; p=0.04)$ and anxiety levels of $\mathrm{BAI}(r=-0.337 ; p=0.027) ; \mathrm{NAA} / \mathrm{Cr}$ ratio was significantly decreased in SLE when compared to healthy controls at all times $(p<0.05)$. It was observed that NAA/Cr ratio was associated with presence of symptoms of anxiety $(p=0.019)$, headache $(p=0.01)$ and correlated with SLICC $(r=-0.359, p=0.004)$ and SLEDAI $(r=-0.275 ; p=0.03)$. The Cho/Cr ratio was associated with headache $(p=0.049)$, dsDNA ( $p=0.04)$ and correlated with illness time $(r=0.215, p=0.002)$. It was also observed that corticoid use was associated with NAA/Cr ratio $(p=0.01)$, Cho/Cr ratio $(p=0.043), G \mid x / C r$ ratio $(p=0.039)$ and the corticoid dose correlated with $\mathrm{GI} / \mathrm{Cr}$ ratio $(r=-0.262 ; p=0.049)$.

\section{CONCLUSION}

We observed significant axonal dysfunction in SLE. Decreased NAA/Cr ratio was associated with symptoms of anxiety, SLICC and SLEDAI. Cho/Cr ratio was associated with illness time and headache. We observed a difference in $\mathrm{Glx} / \mathrm{Cr}$ ratio over and correlated with anxiety and corticoid dose, suggesting brain injury. 